\title{
De Conrad à Simon : l'esthétique du « faire voir »
}

\section{Pierre Schoentjes}

\section{OpenEdition}

\section{Journals}

Édition électronique

URL : https://journals.openedition.org/ccs/446

DOI : $10.4000 /$ ccs.446

ISSN : 2558-782X

\section{Éditeur :}

Presses universitaires de Rennes, Association des lecteurs de Claude Simon

\section{Édition imprimée}

Date de publication : 30 juin 2005

Pagination : 47-57

ISBN : 2914518676

ISSN : 1774-9425

\section{Référence électronique}

Pierre Schoentjes, « De Conrad à Simon : I'esthétique du « faire voir » », Cahiers Claude Simon [En ligne], 1 | 2005, mis en ligne le 20 septembre 2017, consulté le 02 mars 2022. URL : http:// journals.openedition.org/ccs/446 ; DOI : https://doi.org/10.4000/ccs.446 


\title{
De Conrad à Simon : l'esthétique du « faire voir "
}

\author{
Pierre SCHOENTJES*
}

Joseph Conrad est un écrivain envers lequel Claude Simon se reconnaît une dette importante. Au moins depuis la fin des années $60^{1}$ et jusqu'à une date récente, il n'a cessé de rappeler l'estime qu'il porte à l'auteur de Le Nègre du "Narcisse ". Réfléchissant en 1993 à la place qu'occupe le commentaire dans le roman traditionnel, il déclare à Mireille Calle-Gruber :

Stendhal ne montre plus [contrairement aux pages consacrées à Waterloo] : il nous informe et nous dit ce qu'il faut penser (des aubergistes, de Fabrice qui devient " une autre homme ", fait des réflexions "profondes »). Comme l'écrit Conrad dans sa préface au Nègre du "Narcisse " qui constitue le plus magistral réquisitoire contre le roman académique : «[...] l'auteur fait appel à notre crédulité. $»^{2}$

Ce commentaire rappelle certains propos antérieurs de Simon au sujet de la célèbre "Préface " de 1897 au Nègre du "Narcisse ", en

* Université de Gand (Belgique).

${ }^{1}$ Cf. Le Monde hebdo : 6-12 avril 1967.

2 Claude Simon \& Mireille Calle, "L'inlassable réa/encrage du vécu ", in : Claude Simon, chemins de la mémoire. Textes, entretiens, manuscrits, Mireille Calle éd., Grenoble/Quebec, U.G./Le Griffon d'argile, coll. "Trait d'union ", 1993, p. 11. 
particulier ceux exprimés dans le Discours de Stockholm:

Comme le souligne Conrad dans sa préface au Nègre du Narcisse, l'auteur fait appel à notre seule crédulité, car, pour ce qui concerne la « logique » des caractères comme celle des situations, on pourrait en discuter sans fin : tandis qu'Henri Martineau, éminent stendhalien, nous assure que Julien Sorel est prédestiné dès le début du roman Le rouge et le noir à tirer le fatal coup de pistolet sur Madame de Rénal, Émile Faguet, lui, trouve, ce dénouement " plus faux qu'il n'est permis $» . . .3$

À chaque fois Simon dit son admiration pour Conrad et retient de la "Préface " au Nègre du "Narcisse » une donnée manifestement importante à ses yeux et qu'il résume par une citation empruntée : " l'auteur fait appel à notre crédulité ». À considérer attentivement les commentaires de Simon, il apparaît que ce qui semble a priori un simple constat constitue pour lui une des prémisses essentielles à la fois de l'écriture et de la lecture.

Afin de mieux comprendre le sens et la portée de cette citation emblématique, il est nécessaire de se reporter à la lettre de la "Préface » de Joseph Conrad. L'auteur y développe une poétique qui aboutit à une réflexion sur la place de la littérature dans la vie :

Toute œuvre littéraire qui aspire, si humblement soit-il, à s'élever à la hauteur de l'art doit justifier son existence à chaque ligne. Et l'art lui-même peut se définir comme la tentative d'un esprit individuel pour rendre le mieux possible justice à l'univers visible, en mettant en lumière la vérité diverse et une que recèle chacun de ses aspects. C'est l'effort fait pour découvrir dans ses formes, dans ses couleurs, dans sa lumière, dans ses ombres, dans les aspects de la matière et les faits de la vie même, ce qui leur est fondamental, ce qui est durable et essentiel - leur qualité la plus évocatrice et la plus convaincante - la vérité même de leur existence. L'artiste donc, aussi bien que le penseur ou l'homme de science, recherche la vérité pour la mettre en lumière. Séduit par les dessous du monde visible, le penseur s'enfonce dans la région des idées, l'homme de science dans le domaine des faits, dont ils dégagent des vérités pratiques qui conviennent à cette hasardeuse entreprise qu'est notre vie. Ils parlent avec assurance à notre sens commun, à notre intelligence, à

${ }^{3}$ Claude Simon, Discours de Stockholm, Minuit, 1986, p. 18, 19. 
notre désir de paix où à notre inquiétude, fréquemment à nos préjugés, parfois à nos appréhensions, souvent à notre égoïsme, mais toujours à notre crédulité. Et l'on écoute leurs paroles avec respect, car elles ont trait à de graves questions, à la culture de nos esprits ou à la préservation de notre corps, à l'accomplissement de nos ambitions, à la perfection de nos moyens et à la glorification de nos précieux succès.

Il en est tout autrement de l'artiste. [...]

Tout art doit s'adresser d'abord aux sens, et une conception artistique qui s'exprime à l'aide de mots écrits doit s'adresser aux sens, si son intention profonde est d'atteindre la source même de nos émotions. Il lui faut aspirer de toutes ses forces à la plasticité de la sculpture, à la couleur de la peinture, à la suggestion magique de la musique, cet art des arts. Et ce n'est que par une dévotion complète et inébranlable au parfait accord de la forme et de la substance, ce n'est que par un soin incessant apporté au contour et à la sonorité des phrases qu'on peut atteindre à la plasticité et à la couleur, et que la lumière de la suggestion magique peut jouer furtivement à la surface banale des mots, vieux mots épuisés et défigurés par des siècles d'un insouciant usage. [...]

Arrêter pour un temps les mains occupées aux œuvres pratiques de la terre, obliger des hommes absorbés par la vue lointaine de succès matériels à contempler un moment autour d'eux une vision de formes, de couleurs, de lumière et d'ombre ; les faire s'arrêter, l'espace d'un regard, d'un soupir, d'un sourire, tel est le but, difficile et fuyant, et qu'il n'est donné qu'à bien peu d'entre nous d'atteindre. Mais quelquefois par l'effet de la grâce et du mérite, même cette tâche-là peut être accomplie. Et lorsqu'elle l'est - ô merveille ! - toute la vérité de la vie s'y trouve : un moment de vision, un soupir, un sourire, et le retour à un repos éternel. ${ }^{4}$

Juxtaposée aux commentaires de Claude Simon, cette page est à la fois paradoxale et éclairante. Elle est paradoxale parce quà la lecture il apparaît sans ambiguïtés que pour Joseph Conrad ce sont les penseurs et les hommes de science qui font appel à " notre crédulité ». Or, c'est précisément à l'opposé de leur démarche que la

4 Joseph Conrad, Préface au Nègre du "Narcisse" (1898), traduit par R. D’Humières, Gallimard, 1938, incipit ; p. VII ; explicit. 
"Préface " situe celle de l'artiste. Comme le philosophe ou le scientifique, l'écrivain cherche la vérité afin de la mettre en lumière, il n'entend pas, lui, l'imposer en faisant appel à la crédulité. L'art " doit s'adresser d'abord au sens » et montrer une vérité : il ne lui appartient pas de convaincre, un exercice qui nécessite de faire appel à des choses - « sens commun, intelligence, préjugés... » - que l'interlocuteur ne peut pas " voir".

Simon récuse la "crédulité " parce qu'il entend rejeter toute adhésion naïve à une fable. Peut-être d'ailleurs l'auteur a-t-il pu considérer la citation de la "Préface " au Nègre du "Narcisse " comme d'autant plus propice à établir sa position que l'idée de " crédulité " rappelle la célèbre " willing suspension of disbelief " dont Coleridge se servait déjà en 1817 pour caractériser la " poetic faith $»^{5}$.

Pour Simon l'art doit " montrer ", ou plus exactement, et pour reprendre la formule de Joseph Conrad, " faire voir " : " Le but que je m'efforce d'atteindre est, avec le seul pouvoir des mots écrits, de vous faire entendre, de vous faire sentir, et avant tout, de vous faire voir " ${ }^{6}$. Il est clair que s'il entend atteindre ce but, l'art " doit s'adresser d'abord aux sens ", comme le souligne la "Préface ».

Il est manifeste que la page de Conrad constitue une poétique dont s'inspire l'écriture de Simon. On peut d'ailleurs le vérifier sommairement en rappelant quelques éléments saillants du réseau tissé par Les Géorgiques, peut-être le plus visuel des romans de Simon.

L'exergue de cette œuvre est célèbre ; on peut y lire, avant même la préface au dessin, un condensé de la poétique mise en œuvre par l'auteur : "Les climats, les saisons, les sons, les couleurs, l'obscurité, la lumière, les éléments, les aliments, le bruit, le silence, le mouvement, le repos, tout agit sur notre machine et sur notre âme par conséquent. J.J. Rousseau (Les Confessions) ». À relire l'exergue à la

5 Samuel Taylor Coleridge, Biographia Literaria, H.J. Jackson éd., Oxford, 1985, p. 314 .

6 Joseph Conrad, Préface au Nègre du "Narcisse ", op. cit., p. VII. 
suite de la "Préface " au Nègre du "Narcisse », il apparaît qu' elle renvoie non seulement aux préférences littéraires de L.S.M., grand lecteur de Rousseau, mais encore et surtout aux préoccupations esthétiques de Simon.

Comment ne pas lire en effet ces quelques lignes des Confessions comme un renvoi tacite à la page de la "Préface " de Conrad que je viens de rappeler. Les phrases rappellent, plus précisément, le passage où Conrad soutient qu'il appartient à l'art de rendre compte de la diversité du réel : "C'est l'effort fait pour découvrir dans ses formes, dans ses couleurs, dans sa lumière, dans ses ombres, dans les aspects de la matière et les faits de la vie même, ce qui leur est fondamental, ce qui est durable et essentiel - leur qualité la plus évocatrice et la plus convaincante - la vérité même de leur existence ${ }^{7}$. ".

Dans les Géorgiques, Simon cherche lui aussi à tirer profit des ressources esthétiques des autres modes d'expression artistique. Il traite abondamment de peinture (dessin du prologue, portraits de L.S.M....), de sculpture (plasticité et volumes dans le prologue encore, buste de L.S.M.) et de musique (Orfeo de Glück). Mais ce qui est plus important, il sait faire profiter son écriture des moyens techniques de ces différents arts : jeux de lumières et de perspectives propres aux peintres; volumes et mouvements du sculpteur; rythmes et cadences du musicien...

À l'exception - notoire - du prologue, ce n'est jamais l'analyse intellectuelle consciente et active qui est privilégiée par Simon mais au contraire la façon dont les personnages sont livrés, passifs, aux impressions sensorielles. Celles-ci débordent de l'œuvre et submergent le lecteur, réalisant ainsi l'ambition de l'art telle que l'exprime Conrad : "Arrêter pour un temps les mains occupées aux ouvres pratiques de la terre, obliger des hommes absorbés par la vue lointaine de succès matériels à contempler un moment autour d'eux une vision de formes, de couleurs, de lumière et d'ombre ; les faire s'arrêter, l'espace d'un regard, d'un soupir, d'un sourire, tel est le but, difficile et fuyant, et qu’il n'est donné qu'à bien peu d'entre nous d'atteindre ${ }^{8}$. ».

\footnotetext{
7 Ibid.,, p. v, je souligne.
}

8 Ibid., p. XII, je souligne. 
Nous voyons la tempête qui s'abat sur le Narcisse, nous voyons de même le cavalier pris avec son escadron dans l'embuscade. Davantage peut-être que chez Conrad nous assistons chez Simon aux événements de la vie des personnages par le truchement des impressions sensorielles.

Le travail de l'écrivain lui-même est placé sous le signe de ces jeux de couleurs, d'ombre et de lumière : se représentant devant sa table de travail, le narrateur fait observer leur action. Tout au long de sa course, le soleil éclaire différemment les papiers et la main qui les feuillette. Les impressions se succèdent : le matin, "[l]e soleil éclaire d'un jour frisant la main " $(G, 24)$, plus tard " [l]e papier ivoire des feuilles frappé par le soleil renvoie la lumière qui éclaire par en dessous le visage ridé. " (29) ; à mesure que la journée avance " [les] ombres de statues équestres s'étirent sur le pavé selon le même angle que celle projetée sur le cahier par la main." (35). En fin de journée, une mouche vient se poser sur cette main : "Son ombre transparente et monstrueuse distendue en oblique semble emportée par un élan statique [...]" (41) et le soir " [l]a main ridée qui feuillette les registres se trouve maintenant entièrement dans l'ombre et la lumière diffuse luit faiblement sur la surface crêpelée de la peau » (69).

Ces passages introduisent bien évidemment la notion de temps dans le texte, mais on aurait tort d'accorder une importance excessive à la chronologie que ces indications permettent d'établir. $L a$ Route des Flandres mettait déjà en garde contre l'obsession intellectuelle de la chronologie - qui est aussi obsession de la causalité en rappelant qu'au bout du Temps il ne peut y avoir que la Mort. Repassant par l'endroit où gît le cheval, Georges cherche à se situer à partir de la position du soleil, mais il abandonne bientôt : " "Mais nos ombres étaient alors sur notre droite, donc le soleil a maintenant franchi l'axe de la route, donc...", puis cessant de penser, ou plutôt d'essayer de calculer, pensant seulement : "Mais qu'est-ce que ça peut faire ? Qu'est-ce que ça peut bien lui faire maintenant dans l'endroit où il est..." " $(R F, 106)$.

Dans les phrases centrées sur la main comme ailleurs dans le texte des Géorgiques, les sensations signalées dans l'exergue s'adressent dans l'immédiat aux sens, et non dans le temps à l'intelligence. 
L’ « art du regard » de Claude Simon n'est pas celui d'Alain RobbeGrillet dont Jean Bloch-Michel écrivait qu'il " interdit d'interpréter [...] les mouvements ou les paroles de ceux que [1'on] regarde9 ». Le narrateur de Claude Simon ne cesse d'interpréter, d'imaginer, d'accumuler les « comme si "; l'auteur rappelle ainsi à chaque instant sa parenté avec l'idéal artistique de Joseph Conrad en même temps d'ailleurs qu'avec certains aspects de la poétique de Marcel Proust. L'auteur de la Recherche écrit en effet dans le célèbre passage consacré à la métaphore cette phrase dont la lecture ne manque pas de rappeler tant l'exergue de Simon que la "Préface " au Nègre du "Narcisse ":

Une heure n'est pas une heure, c'est un vase rempli de parfums, de sons, de projets et de climats. Ce que nous appelons la réalité est un certain rapport entre ces sensations et ces souvenirs qui nous entourent simultanément - rapport que supprime une simple vision cinématographique, laquelle s'éloigne par là d'autant plus du vrai qu'elle prétend se borner à lui - rapport unique que l'écrivain doit retrouver pour en enchaîner à jamais dans sa phrase les deux termes différents. ${ }^{10}$

L'écriture simonienne s'efforce aussi de saisir ce rapport qui ne peut se passer de l'expérience sensorielle ; l'importance de l'olfactif et du gustatif est réduite en faveur du visuel et du tactile. Pour Simon comme pour Conrad et Proust, l'auteur ne doit pas chercher à expliquer ni à transformer le réel, son rôle se restreint à " faire voir ». Dans certains romans antérieurs aux Géorgiques, on observait une hésitation quant au statut relatif qu'occupaient écriture et expérience. Ainsi on pouvait lire dans Histoire que

... entre le lire dans des livres ou le voir artistiquement représenté dans les musées et le toucher et recevoir les éclaboussures c'est la même différence qui existe entre voir écrit le mot obus et se retrouver d'un instant à l'autre couché cramponné à la terre [...]. (Hist., 152)

Mais dans La Route des Flandres, Georges se montre moins catégorique ; il observe avoir « déjà lu en latin » ce qui lui est arrivé de

\footnotetext{
9 Jean Bloch-Michel, Le Présent de l'indicatif, Gallimard, 1963, p. 16, 17.

${ }^{10}$ Marcel Proust, Le Temps retrouvé, in : À la recherche du temps perdu, Gallimard, "Bibliothèque de la Pléiade », 1989, t. IV, p. 467, 468 ; je souligne.
} 
sorte qu'il avoue ne pas avoir été trop surpris " et même dans une certaine mesure rassuré de savoir que ç’avait déjà été écrit " $(R F$, 100).

Avec le roman de 1981, Simon semble lever l'hésitation : l'écrit ne permet pas de comprendre le vécu, pas plus que le vécu n'est apte à faire comprendre l'écrit : ces deux expériences participent d'une nature différente. Quand le narrateur écrit : "Ils comprennent alors qu'ils sont tombés dans une embuscade et qu'ils vont presque tous mourir ", il relève immédiatement la main car " il se rend compte que [cette phrase] est à peu près incompréhensible pour qui ne s'est pas trouvé dans une situation semblable » $(G, 47)$. L'oncle Charles rappelle à ce propos que même si le narrateur avait vécu une expérience similaire, il ne serait toujours pas question de compréhension, lisons d'intellection : "Je veux dire quand tu seras capable non pas de comprendre mais de sentir certaines choses parce que tu les auras toi-même éprouvées..." (445).

L'auteur ne peut donc que "rapporte[r] dans un roman les circonstances et la façon dont les choses se sont déroulées " $(G, 52)$ et s'efforcer de faire voir le mieux possible au lecteur, par exemple, les " deux cavaliers " (130) protagonistes de La Route des Flandres. Quand il réécrit en deux pages $(312,313)$ l'essai de O. sur la guerre civile en Espagne, le narrateur prend soin de faire précéder son synopsis de ces mots : "il peut voir " tandis qu'à l'autre bout du résumé se lit la déclaration d'intention toute différente de $\mathrm{O}$. : «Il essaie de faire comprendre cela » (314). L'artiste ne s'adresse pas tant à un sens commun ou moral qu'à ce qui découle de l'immédiat perceptible, mais qu'évidemment l'usage de la comparaison ou de la métaphore peut rendre plus présent.

Faire voir, utiliser la force des mots afin de montrer ce qu'ils cachent trop souvent; mettre en œuvre les richesses des impressions sensorielles, tel est le programme de Claude Simon. Un passage de Conrad, extrait de Sous les yeux d'Occident, peut nous aider à mieux comprendre l'ambition de l'écriture simonienne et quelques-uns des problèmes qui s'y rattachent :

Les mots, vous le savez, sont les plus grands ennemis de la réalité. J'ai été pendant de longues années professeur de langues étrangères, occu- 
pation qui finit par devenir fatale pour les qualités d'imagination, d'observation et d'intuition dont un homme ordinaire a pu se sentir doté à un degré quelconque. Le professeur de langue voit infailliblement arriver un moment où le monde ne lui apparaît plus qu'à l'état d'un marché de mots innombrables, et où l'homme fait simplement figure d'animal parlant, peu supérieur en somme à un perroquet. ${ }^{11}$

Rendre au mot sa force visuelle et pour cela le tirer de la banalité. Là doit se concentrer l'effort de celui pour qui l'art n'est ni le lieu de la philosophie, ni celui de l'éthique. Ce n'est qu'à condition de concrétiser cette exigence que l'artiste parviendra, selon les termes de Conrad, à s'adresser à une " part plus profonde » de l'homme.

Si nous avons pu reconnaître que cette part est également celle à laquelle s'adresse Claude Simon, il reste cependant nécessaire de boucler la boucle en montrant comment le programme esthétique se donnait à lire déjà dans l'œuvre de Conrad. On lira donc, en guise de conclusion, une page de Nostromo (1904), un roman dont la thématique liée à la violence présente une parenté avec certains des grands romans simoniens. Dans une petite république sud-américaine, régulièrement en proie à des révolutions, le lecteur assiste à une émeute :

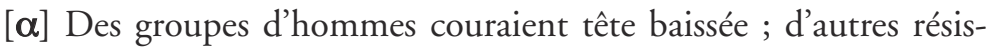
taient ; et le crépitement irrégulier des balles venait par vagues jusqu'à ses oreilles, dans l'air brûlant et immobile. Des hommes isolés, à pied, étaient lancés dans des courses éperdues. Des cavaliers galopaient l'un vers l'autre, décrivaient de conserve un arc de cercle, et se séparaient à toute vitesse. Giorgio en vit tomber un, le cavalier et sa monture disparurent comme s'ils avaient sombré au galop dans l'abîme, et les mouvements de cette fresque vivante ressemblaient aux épisodes d'un jeu violent pratiqué sur la plaine par des nains à pied ou à cheval, hurlant de toute la force de leurs gorges minuscules, au pied de cette montagne qui semblait être une colossale incarnation du silence. Giorgio n'avait encore jamais vu pareille animation sur ce coin de la plaine ; il lui était impossible d'en saisir tous les détails d'un seul regard; il s'abrita les yeux de sa main, quand soudain le tonnerre tout proche d'une multitude de sabots le fit sursauter.

11 Joseph Conrad, Sous les yeux d'Occident (1911), traduit par Ph. Neel, Gallimard, 1920, p. 9. 
[ $\boldsymbol{\beta}]$ Un troupeau de chevaux s'était échappé de l'enclos de la compagnie des chemins de fer. Ils arrivèrent comme une tornade et bondirent par-dessus la voie, renâclant, ruant et hennissant en une masse compacte, houleuse et bigarrée de croupes baies, brunes et grises, les yeux effarés et le cou tendu, le sang aux naseaux, leurs longues queues en oriflammes. Dès qu'ils eurent sauté sur la route, une poussière dense jaillit sous leurs sabots, et à moins de six mètres de Giorgio roula un nuage brunâtre où ne se distinguaient que vaguement des croupes et des encolures, et qui fit trembler le sol sur son passage. ${ }^{12}$

Le narrateur de Conrad cherche à rendre présente la cavalcade sous ses différents aspects, en premier lieu à la vue et à l'ouïe, mais également au toucher, comme en témoigne l'utilisation de mots comme "brûlant» $(\alpha)$ et " trembler» $(\beta)$. Le désordre de la cavalcade mouvante est organisé par l'écriture, qui lui confere une forme en distinguant individus et masses ou encore en rappelant certaines figures géométriques, ainsi l' "arc de cercle» $(\alpha)$. La vision est imprécise - "ne se distinguaient que vaguement» $(\beta)$ - et fragmentée : pour Giorgio qui assiste aux troubles, il est en effet « impossible d'en saisir tous les détails d'un seul regard" $(\alpha)$. Le narrateur est soucieux de faire justice aux effets de profondeur, aux éloignements et aux rapprochements - "vague ", "l'un vers l'autre " $(\alpha)$. Cette attention pour la perspective est soulignée par la référence aux arts plastiques, ainsi qu'en témoigne l'évocation de la "fresque vivante » $(\alpha)$.

De manière plus générale, le passage se structure en soulignant les jeux d'oppositions, d'ailleurs très simoniens : entre le mobile et l'immobile - "les cavaliers" vs "la montagne » $(\alpha)$-, le bruit et le silence - " hurlant " vs " silence " $(\alpha)$ - ou encore l'apparition et la disparition - "ils arrivèrent" $(\beta)$ vs "le cavalier et sa monture disparurent " $(\alpha)$. Le regard qui observe la scène est lui-même contrasté puisque la description dramatique de la violence - tirs, chute d'une homme $(\alpha)$ - est suivie d'une version qu'on pourrait nommer parodique ou grotesque mais qui s'inscrit certainement dans un registre

12 Joseph Conrad, Nostromo (1904), traduit par P. Le Moal, in : Euvres II, Gallimard, "Bibliothèque de la Pléiade », 1985, p. 581. 
ludique - « jeu violent pratiqué sur la plaine par des nains à pied ou à cheval» $(\alpha)$.

Le champ métaphorique fait appel à l'univers de la mer "vague " $(\alpha)$, "tornade ", " houleuse " $(\beta)$ - pour décrire le mouvement ; l'élément liquide n'est toutefois jamais souligné pour luimême ni exploité plus avant. On observe en outre que le narrateur confere une forte charge symbolique au décor de la scène en soulignant la présence massive de " cette montagne qui semblait être une colossale incarnation du silence" $(\alpha)$.

Au-delà même du thème de la course de chevaux, la parenté avec Simon apparaît clairement, même si les différences entre les deux écritures ne sont pas moins manifestes. Si les forts contrastes et les jeux de la métaphore rappellent Simon, la ponctuation de Conrad reste à l'évidence beaucoup plus traditionnelle. Pour prendre l'exacte mesure de l'héritage et de ses limites, il faudrait toutefois une étude beaucoup plus détaillée, qu’il est impossible d'envisager ici.

On invitera donc les lecteurs de Simon à retourner plus souvent vers les romans et les nouvelles de Conrad. Ce retour se justifie pleinement : non seulement en raison de l'influence exercée par Conrad, mais encore parce que la connaissance de Simon renouvelle le plaisir qu'il y a à lire cette autre œuvre dans laquelle l'esthétique du " faire voir " tient une si grande place. 